\title{
Schneller zum richtigen Antibiotikum
}

\section{Adrian Ritter}

Freischaffender Journalist

Wie findet man schnellstmöglich das wirksame Antibiotikum für eine Patientin oder einen Patienten? Das Start-up «Resistell» hat einen neuartigen Test für Antibiotikaresistenzen entwickelt.

Wer sich bewegt, hat verloren. Was bei einem beliebten Kinderspiel gilt, trifft auch im Reich der Mikroben zu. Mit einer neuartigen Testmethode misst das Start-up «Resistell» die feinsten Bewegungen von Mikroben. Solange sich Bakterien bewegen, verraten sie damit auch, dass sie noch leben. Werden nun verschiedene antibiotische Wirkstoffe der Probe zugefügt, lässt sich die wirksame Substanz daran erkennen, dass die Bewegung der Mikroben stoppt.

Mit diesem eleganten Prinzip krempelt Resistell das bisherige Vorgehen um. Herkömmliche Verfahren messen das Bakterienwachstum in Kulturen, was deutlich länger dauert. "Musste man vorher ein bis zwei Tage auf das Testergebnis warten, wissen wir jetzt innerhalb von ein paar Stunden, welches Antibiotikum wirkt», sagt Dr. Danuta Cichocka, Mikrobiologin und CEO von Resistell. Bei der Weiterentwicklung von Resistell soll in Zukunft ein zusätzlicher Vorteil der Methode nutzbar gemacht werden: Die Intensität der mikrobiellen Bewegung gibt auch gleich einen Hinweis, wie hoch die Dosis der Antibiotikagabe sein muss.

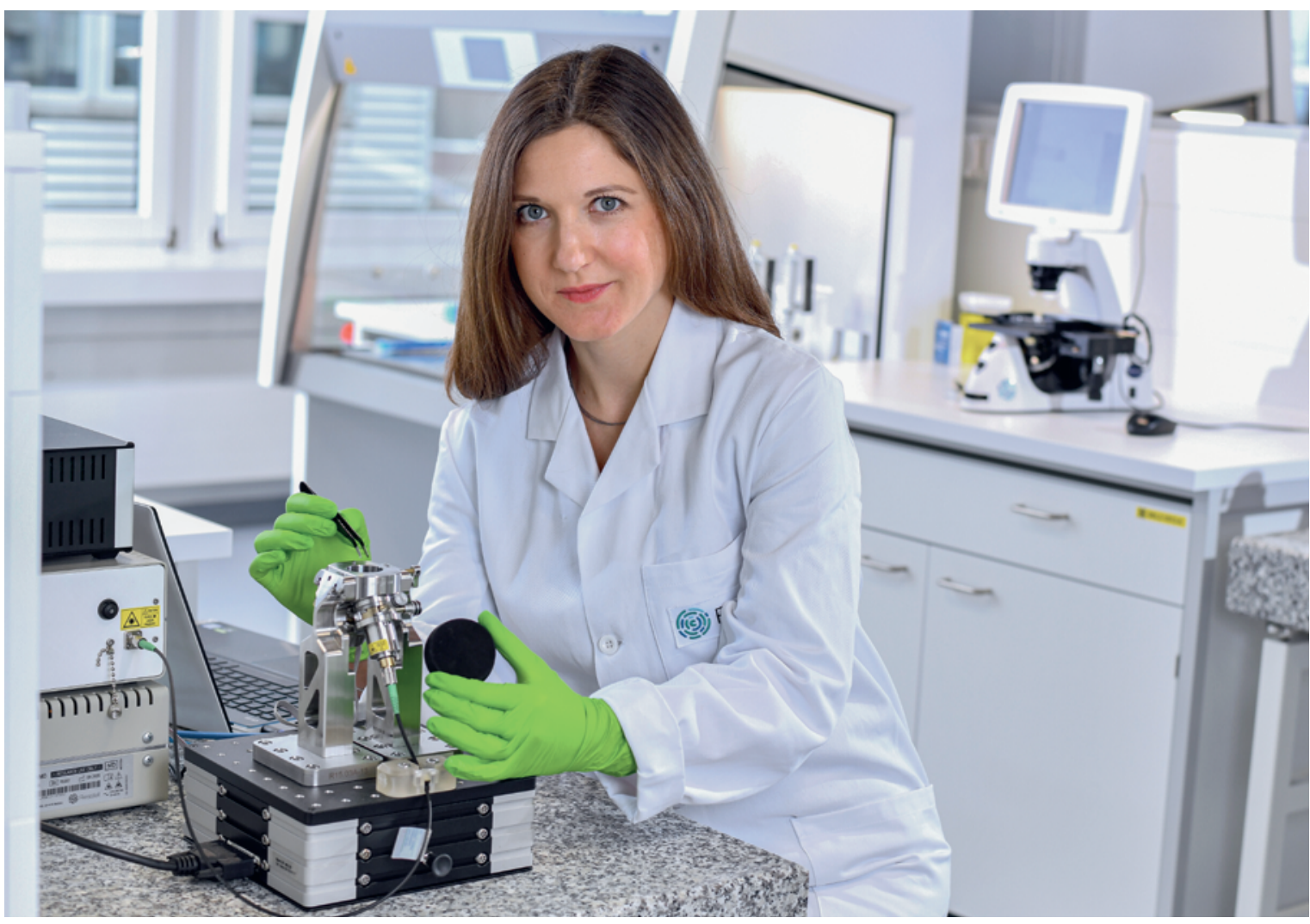

«Musste man vorher ein bis zwei Tage auf das Testergebnis warten, wissen wir jetzt innerhalb von ein paar Stunden, welches Antibiotikum wirkt», sagt Dr. Danuta Cichocka, Mikrobiologin und CEO von Resistell. 


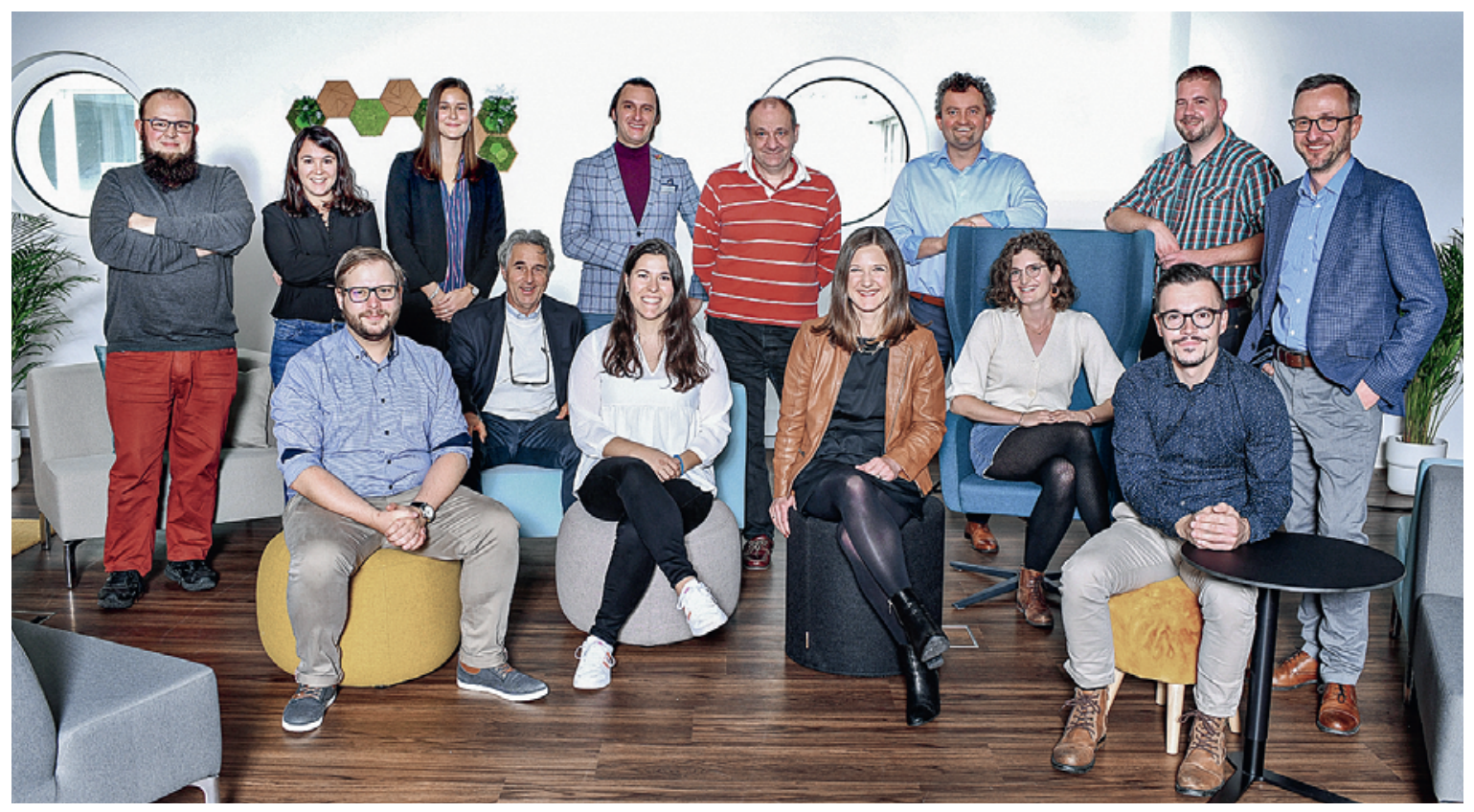

Das Start-up Resistell besteht aus einem Team mit Expertise in Mikrobiologie, Ingenieurwesen, Datenwissenschaft und Entrepreneurship. Unten, dritte von rechts, ist CEO Danuta Cichocka.

\section{Oberflächen abtasten}

Das Start-up Resistell wurde 2018 gegründet und besteht aus einem Team mit Expertise in Mikrobiologie, Ingenieurwesen, Datenwissenschaft und Entrepreneurship. Die Grundlagen für das neuartige Antibiotika-Testverfahren legte eine interdisziplinäre Forschungsgruppe um Physikprofessor Giovanni Dietler und Mediziner Sandor Kasas an der ETH Lausanne. Die Forschenden gingen für ihre neue Testmethode von der Rasterkraftmikroskopie aus - ein Verfahren, das die Abtastung von Oberflächen und die Messung selbst atomarer Kräfte erlaubt - und entwickelten diese weiter.

Resistell liefert Resultate dort schnell, wo wenige Stunden über Leben und Tod entscheiden können - etwa beim Antibiotikaeinsatz bei einer Sepsis. Dabei will Resistell nicht nur den Patientinnen und Patienten helfen, sondern auch das Problem der Antibiotikaresistenzen entschärfen helfen: «Insbesondere in lebensbedrohlichen Situationen setzen Ärztinnen und Ärzte heute schnell Breitbandantibiotika ein, weil die Zeit fehlt, eine spezifisch wirksame Substanz zu suchen. Breitbandantibiotika sollten aber die letzte Therapieoption sein, denn sie haben mehr Nebenwirkungen", sagt Cichocka. Setzen die Ärztinnen und Ärzte andererseits auf ein spezifisches Antibiotikum, das aller- dings zu schwach wirkt, besteht die Gefahr, dass sich Resistenzen bilden - und auch Breitbandantibiotika später nicht mehr wirksam sind.

\section{Corona verschärft das Problem}

Wegen der Corona-Pandemie verschärft sich das Problem der Antibiotikaresistenzen weltweit noch, ist Cichocka überzeugt. Denn Antibiotika werden noch häufiger eingesetzt, um bei Covid-19-Patienten bakterielle Ko-Infektionen zu verhindern.

Resistell hat erfolgreiche Finanzierungsrunden hinter sich. Jetzt sollen Anfang 2021 klinische Studien am Universitätsspital Lausanne und später an zwei Universitätsspitälern in Deutschland und Dänemark beginnen. Zuerst wollen die Forschenden die Zulassung des neuen Verfahrens zur Behandlung von Sepsis erlangen, anschliessend sollen Studien mit Erregern von Lungeninfektionen und sexuell übertragbaren Krankheiten folgen. CEO Danuta Cichocka ist zuversichtlich, dass Resistell Ende 2022 auf den Markt kommen kann.

Mehr Infos unter: www.resistell.com

Bildnachweis

(c) Pino Covino/Resistell 